\title{
Refractory Alveolar Rhabdomyosarcoma
}

National Cancer Institute

\section{Source}

National Cancer Institute. Refractory Alveolar Rhabdomyosarcoma. NCI Thesaurus.

Code C142880.

Alveolar rhabdomyosarcoma that does not respond to treatment. 